\title{
Two-level system in a one-mode quantum field: numerical solution on the basis of the operator method
}

\author{
I D Feranchuk, L I Komarov and A P Ulyanenkov \\ Department of Theoretical Physics, Belarussian State University, 4 Fr. Skariny av., 220080 \\ Minsk, Republic of Belarus \\ Received 29 August 1995, in final form 18 January 1996
}

\begin{abstract}
Accurate eigenvalues and eigenfunctions of a two-level system interacting with a one-mode quantum field are calculated numerically. A special iteration procedure based on the operator method permits one to consider the solution within a wide range of the Hamiltonian parameters and to find the uniformly approximating analytical formula for the eigenvalues. Characteristic features of the model are considered, such as the level intersections, the population of the field states and the chaotization in the system through the doubling of the frequencies.
\end{abstract}

\section{Introduction}

The model of a two-level system in a one-mode quantum field (TLS) is one of the simplest but most widespread and effective models used for consideration of the qualitative characteristics of the interaction between a quantum system and a resonant external field (see, for example, Allen and Eberly 1975 and papers cited therein). It is of great interest both as a mathematical problem and as a good physical model for consideration of non-adiabatic transitions (Reik et al 1982), squeezed states (Meystre and Zubairy 1982), the quantum chaotic system (Graham and Hoehnerbach 1984) and a series of other effects.

The most popular simplification of the TLS Hamiltonian is connected with the JaynesCummings (1963) model (JCM) which is exactly solvable due to the so-called rotating-wave approximation (RWA). But strict analysis of the JCM validity is not usually considered in concrete applications and the range of the system parameters where the results are correct remains uncertain. Moreover, the exact isolated solutions for TLS were found by several authors (e.g. Kus and Lewenstein 1986) and it was proved that the JCM did not describe peculiarities of the accurate energy spectrum in dependence on the atom-field coupling constant.

Therefore it is of great interest to analyse the accurate numerical solution of the TLS problem within a wide range of the Hamiltonian parameters in comparison with the JCM results. Such studies are useful for determining the limits of validity of the JaynesCummings model as well as for describing physical systems with a rather large coupling constant (e.g. the interaction of condensed matter with high-power laser radiation or the processes occurring in long-wave coherent radiation).

Besides, we hope that a fast and effective algorithm for numerical solution of the Schrödinger equation for the TLS Hamiltonian with arbitrary coupling constant and quantum numbers would allow clarification of the status of the conjecture of Reik et al $(1986,1987)$ about the existence of an exact solution to the Schrödinger equation with a TLS Hamiltonian by known functions. 
There is the continued-fraction algorithm used by a number of the authors for numerical studies of the spectrum and the time evolution of the system (e.g. Lais and Steimle 1990). But this method is not particularly effective for a large coupling constant and quantum numbers because of the influence of the exponentially diverging solution of the Schrödinger equation.

In this paper we use the operator method (OM) for the numerical and analytical analysis of the TLS problem. This method proved to be very efficient for the description of various quantum systems (Feranchuk and Komarov 1982, 1984, 1995). It is essential that the qualitative peculiarities of an energy spectrum can be described even in the oM zeroth approximation and the consequent approximations uniformly converge to the exact values in the whole range of system parameters. We shall see (section 2), that the OM algorithm permits one to find the eigenvalues and eigenfunctions of the TLS Hamiltonian with any necessary accuracy and to analyse various characteristics of the system with almost the same efficiency as on the basis of the analytical solution. It is found that the JCM results coincide qualitatively with the accurate ones only in the range of small coupling constant and when low levels are excited. It will also be shown (section 3) that the om zeroth approximation for the TLS problem leads to analytical formulae which uniformly fit the accurate energy spectrum for any parameters of the system.

In section 4 we shall consider some examples of application of the OM numerical solution to analysis of the time evolution of the wavepackets in the TLS model. It will be shown that a number of characteristic features of the TLS which become apparent due to an exact solution of the problem essentially extends the possibilities of the model for description of various physical phenomena.

\section{Numerical solution of the Schrödinger equation}

The well known Hamiltonian of the considered model has the simple dimensionless form

$$
\hat{H}=\frac{1}{2} E \sigma_{3}+a^{+} a+f\left(\sigma_{+}+\sigma_{-}\right)\left(a+a^{+}\right)
$$

where $a^{+}$and $a$ are the photon creation and annihilation operators, $E$ is the atomic level separation energy, $f$ is the atom-field coupling constant proportional to the dipole moment of the transition, $\sigma_{3}, \sigma_{ \pm}=\frac{1}{2}\left(\sigma_{1} \pm \mathrm{i} \sigma_{2}\right)$ are the usual Pauli matrices with the commutation relations

$$
\left[\sigma_{i}, \sigma_{j}\right]=2 \mathrm{i} \varepsilon_{i j k} \sigma_{k}
$$

and the energy units are chosen such that the photon frequency equals 1.

Recall that the Hamiltonian (1) leads to the exact solvable JCM if the 'counter-rotating terms' $\sigma_{+} a^{+}, \sigma_{-} a$ are omitted.

The exact integral of motion ('combined parity') is assumed to exist in the system; it can be written in the form (the representation corresponding to the Hamiltonian (1)):

$$
\hat{P}=\sigma_{3} \hat{S}=\sigma_{3} \mathrm{e}^{\mathrm{i} \pi a^{+} a} .
$$

Thus, the exact state vector of the system depends on two quantum numbers and it is the joint solution to the following equations:

$$
\hat{H}\left|\Psi_{n p}\right\rangle=\mathcal{E}_{n p}\left|\Psi_{n p}\right\rangle \quad \hat{P}\left|\Psi_{n p}\right\rangle=p\left|\Psi_{n p}\right\rangle .
$$

Here the numbers $p= \pm 1$ define the parity and $n=0,1,2, \ldots$ are the energy quantum numbers for the steady-state eigenvalues $\mathcal{E}_{n p}$.

Accordingly to the OM prescription (Feranchuk and Komarov 1984) one has to use a complete set of the basic vectors depending on arbitrary parameters which take into account 
the variation of the system states due to interaction. In the case considered the atom-field coupling leads to a shift of equilibrium position of the field oscillators. This shift can be described by means of the following canonical transformation in the operator form:

$$
\begin{array}{ll}
a=-u+b & a^{+}=-u+b^{+} \\
b=\hat{R}^{-1} a \hat{R} & \hat{R}=\mathrm{e}^{u\left(a^{+}-a\right)}=\mathrm{e}^{-u^{2} / 2} \mathrm{e}^{u a^{+}} \mathrm{e}^{-u a}
\end{array}
$$

where $b^{+}$and $b$ are the new creation and annihilation operators, parameter $u$ will be defined later.

Then the relevant basic set can be chosen by means of eigenfunctions of the operators $\hat{n}=b^{+} b$ and $\sigma_{1}$

$$
\begin{aligned}
& \left|\Phi_{n s}\right\rangle=|n, u\rangle \chi_{s} \\
& \hat{n}=n|n, u\rangle \quad \sigma_{1} \chi_{s}=s \chi_{s} \quad(s= \pm) .
\end{aligned}
$$

The state $|n, u\rangle$ corresponds to the $n$-quantum excitation of the field coherent state and connects with the photon vacuum $|0\rangle$ as follows:

$$
|n, u\rangle=\frac{\left(a^{+}+u\right)^{n}}{\sqrt{n !}} \sum_{k=0}^{\infty} \frac{u^{k}}{k !}\left(a^{+}\right)^{k}|0\rangle \mathrm{e}^{-u^{2} / 2} .
$$

The amplitude $u$ of the classical component of the field to be determined actually defines the choice of the presentation for the wavefunction of the system stationary states.

The functions $\chi_{s}$ are the following linear combinations of the atom ground and excited states:

$$
\chi_{ \pm}=\frac{1}{\sqrt{2}}\left(\chi_{\uparrow} \pm \chi_{\downarrow}\right) .
$$

The transformation of (4) results to the presentation of (3) in the following form:

$$
\begin{aligned}
& {\left[\frac{1}{2} E \sigma_{3}+u^{2}-2 u f \sigma_{1}+\left(-u+f \sigma_{1}\right)\left(b+b^{+}\right)+b^{+} b\right]\left|\Psi_{n p}\right\rangle=\mathcal{E}_{n p}\left|\Psi_{n p}\right\rangle} \\
& \sigma_{3} \mathrm{e}^{-2 u^{2}} \mathrm{e}^{2 u b^{+}} \mathrm{e}^{\mathrm{i} \pi b^{+} b} \mathrm{e}^{2 u b}\left|\Psi_{n p}\right\rangle=p\left|\Psi_{n p}\right\rangle
\end{aligned}
$$

and the state vector is expanded in the following series of basic set functions:

$$
\left|\Psi_{n p}\right\rangle=\sum_{k=0}^{\infty} \sum_{s= \pm} C_{k s}^{n p}|k, u\rangle \chi_{s} .
$$

Then the algebraic recurrence relations for coefficients $C_{k+}^{n p}$ and $C_{k-}^{n p}$ can be found as

$$
\begin{aligned}
& \left(k+u^{2}-2 u f-\mathcal{E}_{n p}\right) C_{k+}^{n p}+(f-u)\left(\sqrt{k} C_{k-1,+}^{n p}+\sqrt{k+1} C_{k+1,+}^{n p}\right)+\frac{1}{2} E C_{k-}^{n p}=0 \\
& C_{k-}^{n p}=p \sum_{m} S_{k m}(u) C_{m+}^{n p}
\end{aligned}
$$

where

$$
\begin{aligned}
& S_{k m}(u)=S_{m k}(u) \\
& S_{k m}(u)=(-1)^{m} \mathrm{e}^{-2 u^{2}} \sqrt{\frac{m !}{k !}}(2 u)^{k-m} L_{m}^{k-m}\left(4 u^{2}\right) \quad k \geqslant m .
\end{aligned}
$$

Here $L_{n}^{\alpha}(x)$ are the Laguerre polynomials (HTF 1953).

The equation for $C_{k-}^{n p}$ also follows from (7), but it is the linear combination of equations (9) when $\mathcal{E}_{n p}$ coincides with the exact eigenvalue. Further numerical calculations show that the connection between $C_{k-}^{n p}$ and $C_{k+}^{n p}$ due to the exact integral of motion $\hat{P}$ is more important for convergence of the OM series than the connection between these coefficients due to equation (7). 
The most effective algorithm for calculation of the oM successive approximation is based on the simple iterations within the system of equations for an unknown eigenvalue and coefficients of the wavefunction expansion. This scheme provides that the diagonal part of the total Hamiltonian is taken into account exactly in every iteration order (Feranchuk et al 1995). Applying this algorithm to equations (9) we arrive at the following recurrence relations for the successive approximations to the accurate values $\mathcal{E}_{n p}$ and $C_{k+}^{n p}$ :

$$
\begin{aligned}
\mathcal{E}_{n p}(t)=n+u^{2} & -2 u f+\frac{1}{2} p E S_{n n}(u) \\
+ & (f-u)\left(\sqrt{n} C_{n-1,+}^{n p}(t-1)+\sqrt{n+1} C_{n+1,+}^{n p}(t-1)\right) \\
& +\frac{1}{2} p E \sum_{m(\neq n)} S_{n m}(u) C_{m+}^{n p}(t-1) \\
C_{k+}^{n p}(t)=\delta_{k n}- & \left(1-\delta_{k n}\right)\left[k+u^{2}-2 u f+\frac{1}{2} p E S_{k k}(u)-\mathcal{E}_{n p}(t-1)\right]^{-1} \\
& \left\{(f-u)\left[\sqrt{k} C_{k-1,+}^{n p}(t-1)+\sqrt{k+1} C_{k+1,+}^{n p}(t-1)\right]\right. \\
+ & \left.\left.\frac{1}{2} p E \sum_{m(\neq k)} S_{k m}(u) C_{m+}^{n p}(t-1)\right]\right\} .
\end{aligned}
$$

and the values $\mathcal{E}_{n p}$ and $C_{k+}^{n p}$ are defined as the limits of the sequences

$$
\mathcal{E}_{n p}=\lim _{t \rightarrow \infty} \mathcal{E}_{n p}(t) \quad C_{k+}^{n p}=\lim _{t \rightarrow \infty} C_{k+}^{n p}(t) .
$$

One can use the OM zeroth-order approximation in order to find the initial elements of the sequences (Feranchuk and Komarov 1982)

$$
C_{k+}^{n p}(0)=\delta_{n k} \quad \mathcal{E}_{n p}(0)=n+u^{2}-2 u f+\frac{1}{2} p E S_{n n}(u) .
$$

By definition the exact eigenvalues do not depend on the parameter $u$ which is related to the choice of the wavefunction representation only and the accurate numerical calculations confirm this statement. But the rate of convergence of the sequences (13) depends on this value (Feranchuk et al 1995) and it proves to be maximal when

$$
u=f
$$

This choice is optimal for the om zeroth approximation as well (see below, section 3).

The iteration scheme described above permits one to find the solution to the Schrödinger equation for the TLS with any necessary accuracy within the entire range of the Hamiltonian parameters. In this sense we shall consider this solution as an exact one and it proves to be as effective for the analysis of the system characteristics as an analytical solution.

Let us consider some features of the exact solution which are qualitatively different from the results obtained in the limits of the Jaynes-Cummings model. Figures $1(a)$ and (b) compare the accurate energy spectrum of the system with its asymptotic approximations: the rotating-wave approximation in the case $f \ll 1$ and $\mathcal{E}_{n p} \simeq n-f^{2}$ in the limit of strong coupling. One can see that the intersections of the levels with different parity theoretically described earlier (Kus and Lewenstein 1986) lead to the formation of peculiar 'plaits' on the diagram of the levels. The real spectrum structure can be approximated by the spectrum of the JCM only in the range of a sufficiently small coupling constant until the first intersection of the levels with different parity. The width of this range decreases for highly excited states of the system proportionally to $1 / \sqrt{n}$. Figures $1(c)$ and $(d)$ illustrate this statement and show that our numerical solution is effective for any quantum numbers.

Figure 2 shows the contribution of various harmonics of the field in the formation of the accurate stationary states. We recall that in the JCM limits the $n$th state of the system is 

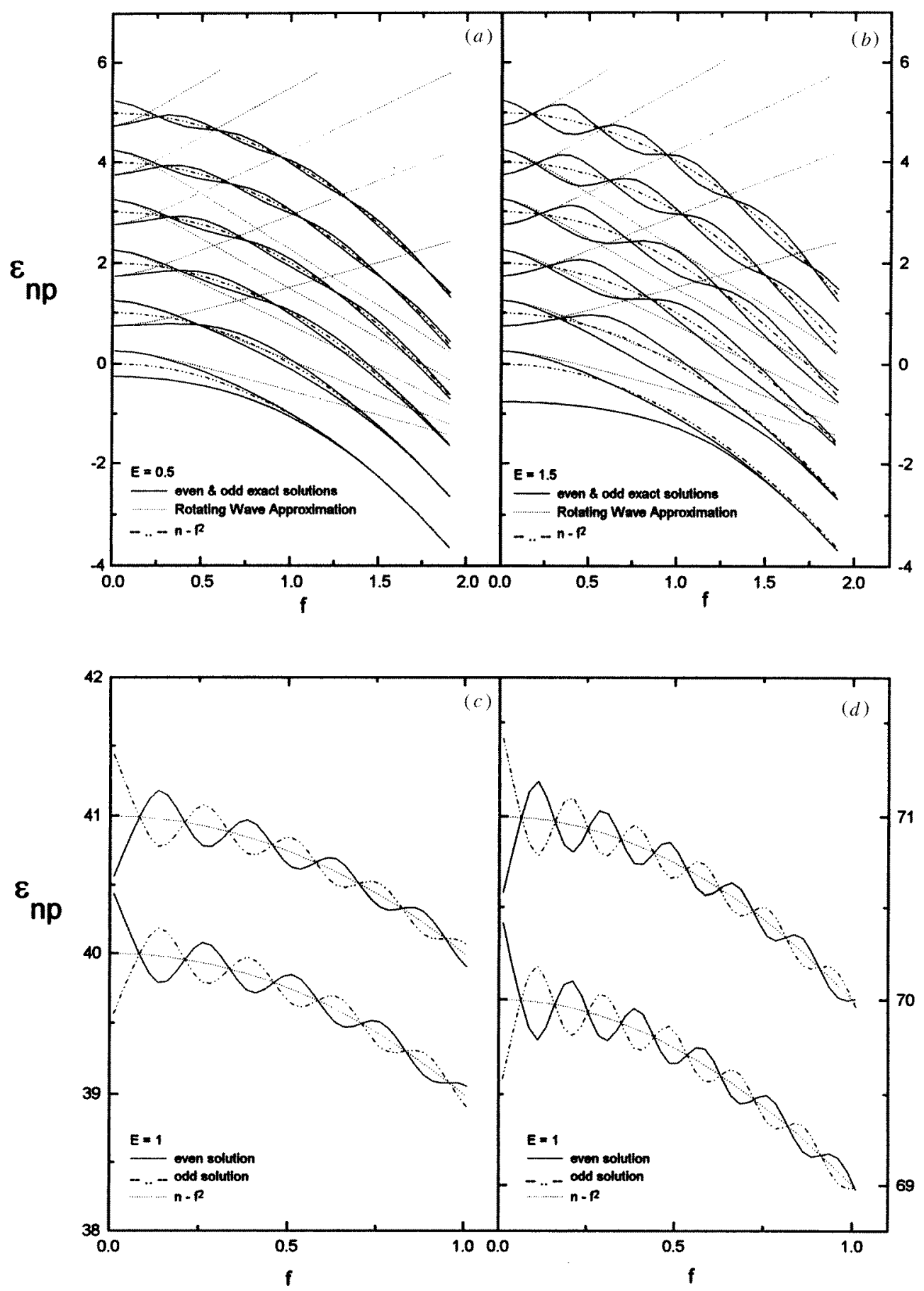

Figure 1. $(a),(b)$ Exact and approximate eigenvalues of the TLS Hamiltonian as the functions of the coupling constant and the separation energy; $(c),(d)$ highly excited states of the TLS.

the superposition of two field states only. But one can see from figure 2 that, for example, actually quite a lot of field quanta contribute to the formation of the first system excited 


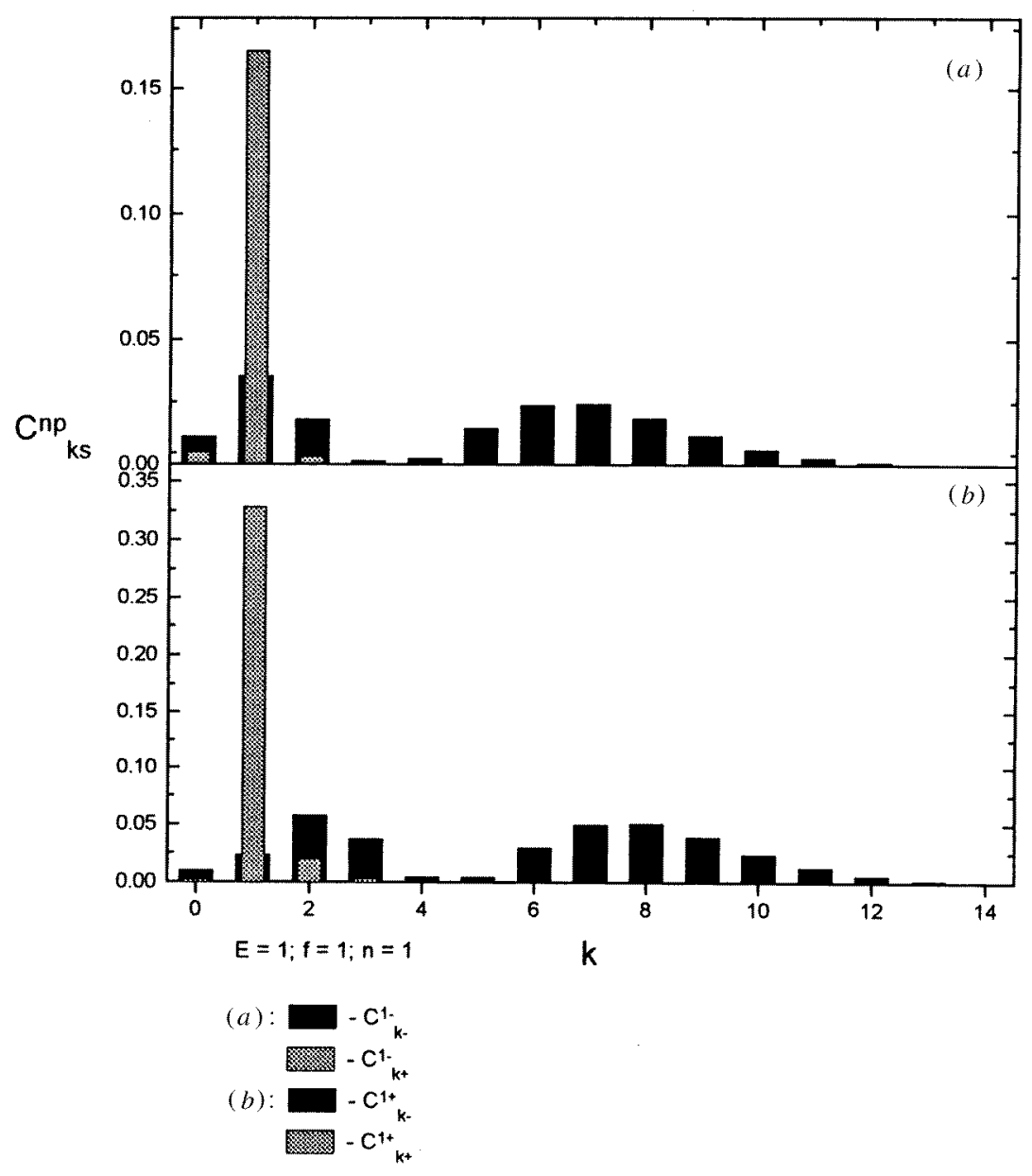

Figure 2. Coefficients of the series on the basic set functions for the first excited $(a)$ even and (b) odd states of the TLS.

state even for a coupling constant of $f \simeq 1$.

We also verified the conjectured analytical solution by Reik et al $(1986,1987)$ for these parameters considered in their paper. Unfortunately this very important solution is fundamentally connected to an inconvenient calculation of spherical functions. Therefore we intend to compare this solution in detail with the exact numerical results in a separate paper.

Some other accurate results will be discussed later (section 4) when the time evolution of the system will be considered.

\section{Zeroth approximation of the operator method}

It is possible to deduce the approximate but analytical formulae which interpolate energy and other characteristics of the TLS uniformly within the entire range of the coupling constant and quantum number variation. The solution to this problem can be obtained in the OM zeroth approximation. 
In accordance with the OM algorithm (Feranchuk et al 1995) one has to take into account only the diagonal part of the full Hamiltonian with respect to the considered basic set in order to find the OM zeroth-order approximation. To put this into the matrix equations (7) let us expand the state vector $\left|\Psi_{n p}\right\rangle$ in the eigenfunctions of the matrix $\sigma_{1}$

$$
\left|\Psi_{n p}\right\rangle=\left|\varphi_{n p+}\right\rangle \chi_{+}+\left|\varphi_{n p-}\right\rangle \chi_{-}
$$

and exclude the 'energy spin' variables.

Then the effective Schrödinger equation is

$$
\hat{H}_{\text {eff }}\left|\varphi_{n p+}\right\rangle=\left[u^{2}-2 u f+b^{+} b+(f-u)\left(b+b^{+}\right)+\frac{1}{2} p E \hat{S}\right]\left|\varphi_{n p+}\right\rangle
$$

where $\hat{S}$ is the operator with matrix elements defined in equation (10).

In accordance with the generalized form of the OM considered by Feranchuk et al (1987) the diagonal form of the Hamiltonian can be extracted for linear combinations of the vectors from the basic set which take into account the most essential properties of the exact wavefunctions. In the case considered such a peculiarity is defined by the degeneration (for $f=0$ and $u=0)$ of the states with the quantum numbers $n$ and $n+q\left(q=p(-1)^{n}\right)$ when $E=1$, and can be described by means of the following 'correct linear combinations'

$$
\left|\varphi_{n p+}^{(0)}\right\rangle=A|n, u\rangle+B|n+q, u\rangle
$$

with constant coefficients $A$ and $B$.

Certainly the exact eigenvalues do not depend on a choice of the parameter $u$, which defines only the wavefunctions representation. But just this artificial parameter ensures 'flexibility' of the OM zeroth-order approximation for various coupling constants and there are several ways to choose the optimal value for this parameter (Feranchuk et al 1995).

It proves that the optimal value $u$ for the effective Hamiltonian (16) is very simple, $u=f$, which permits one to turn into zero the main part of the operator which defines the transitions between the nearest states. Applying the state vector (17) to equation (16) leads to the following analytical formula for the stationary state energies in the OM zeroth approximation:

$$
\begin{aligned}
\mathcal{E}_{n p}^{(0)}=n+\frac{1}{2} q & -f^{2}+\frac{1}{4} E q(-1)^{n}\left(S_{n n}(f)+S_{n+q, n+q}(f)\right) \\
& -\frac{1}{2} q \sqrt{\left[1-\frac{1}{2} E(-1)^{n}\left(S_{n n}(f)-S_{n+q, n+q}(f)\right)\right]^{2}+E^{2} S_{n, n+q}^{2}(f)} .
\end{aligned}
$$

Similar to other applications of the om formula, equation (19) proves to be uniformly suitable because it reproduces the exact asymptotic behaviour of the function $\mathcal{E}_{n p}$ in the limiting cases $f \gg 1, f \ll 1$ and describes rather accurately the quantitative peculiarities of the energy spectrum for the intermediate values of the coupling constant (see figure 3 ).

Indeed, one can obtain the following formula for $f \ll 1$ :

$$
S_{n n}(f) \approx(-1)^{n} \quad S_{n, n+q}(f) \approx(-1)^{n+\frac{1}{2}(1+q)} 2 f \sqrt{n+\frac{1}{2}(1+q)}
$$

and (for $|1-E| \leqslant f$ )

$$
\mathcal{E}_{n p}^{(0)} \approx n+\frac{1}{2} q-\frac{1}{2} q \sqrt{(1-E)^{2}+4 f^{2}\left[n+\frac{1}{2}(1+q)\right]}
$$

which coincides with the results of the Jaynes-Cummings model.

In the opposite limit case $f \gg 1$ the exponentially small terms in formula (19) can be omitted and it leads to the simple expression

$$
\mathcal{E}_{n p}^{(0)} \approx n-f^{2}
$$

which is asymptotically exact. 


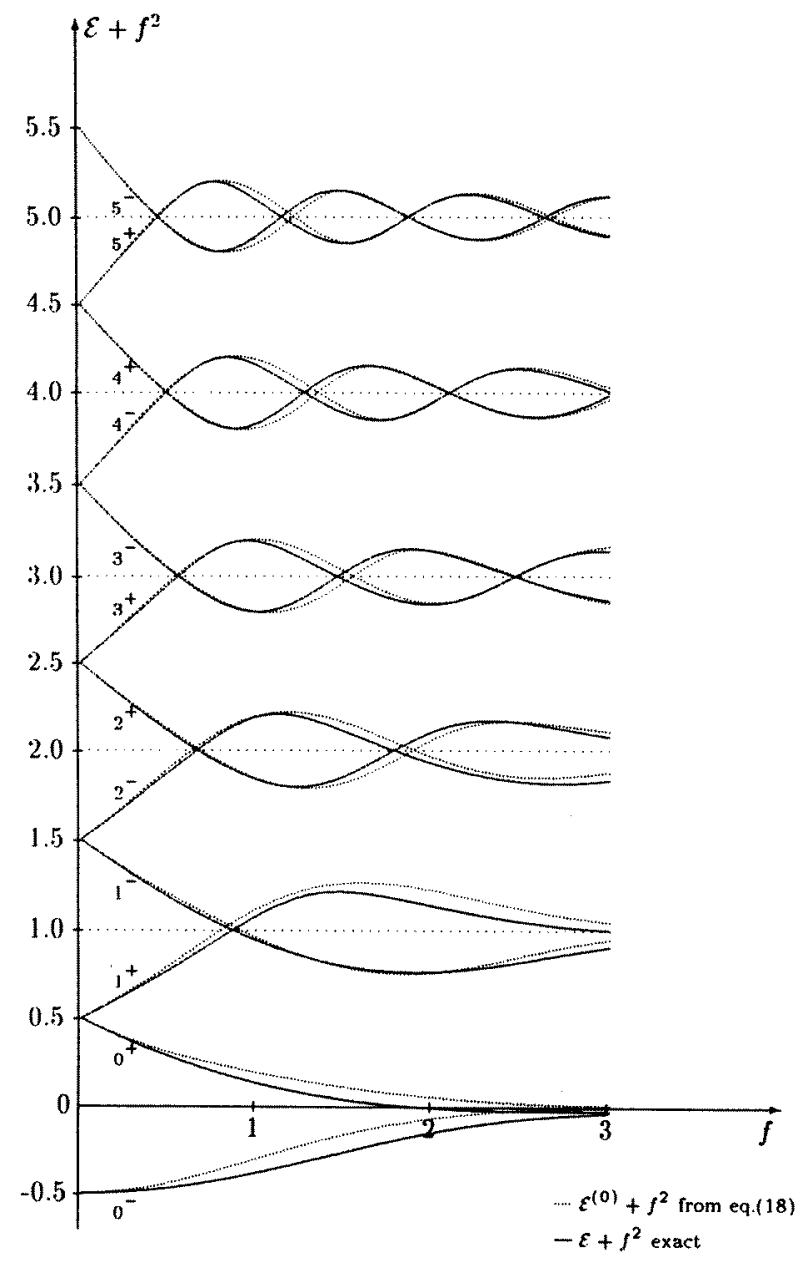

Figure 3. Comparison of the exact eigenvalues with the om zeroth approximation.

A simpler formula (13) may be used as the zeroth approximation taking into account the fact that the degeneration of levels is significant only in the domain of small $f$

$$
\mathcal{E}_{n p}^{(0)}=n-f^{2}+\frac{1}{2} q E \mathrm{e}^{-2 f^{2}} L_{n}\left(4 f^{2}\right) \quad q=p(-1)^{n} .
$$

As could be seen in section 2, the characteristic feature of the energy spectrum for the system considered is the intersection of the levels with the same quantum number $n$ and different parity. Equation (18) (or (22)) shows that the oM zeroth-order approximation for the spectrum has the same property (see figure 3 ).

The approximate estimation for roots of the equation for $f^{2}$

$$
\mathcal{E}_{n, 1}(f)=\mathcal{E}_{n,-1}(f)
$$

with $\mathcal{E}$ from (22) can be found by means of the simple formula

$$
L_{n}\left(4 f^{2}\right)=0 .
$$

In accordance with the exact results (Kus and Lewenstein 1986) the $L_{n}\left(4 f^{2}\right)$ is the polynomial of degree $n$ with alternating terms in relation to $f^{2}$. As for example solutions 
of equation (24) for $n=1$ and $n=2$ are written as

$$
f_{1}^{2}=\frac{1}{4}, n=1 \quad f_{1,2}^{2}=\frac{1}{4}(2 \pm \sqrt{2}), n=2
$$

and can be compared with analogous accurate values (Kus and Lewenstein 1986)

$$
f_{1}^{2}=\frac{1}{4}\left(1-\frac{1}{4} E^{2}\right) \quad f_{1,2}^{2}=\frac{1}{4}\left(2-\frac{3}{16} E^{2} \pm \sqrt{2-\frac{1}{8} E^{2}+\frac{1}{256} E^{4}}\right) .
$$

So the simple equation (24) permits one to find the characteristic points of the spectrum with accuracy $\sim 25 \%$ in the most interesting resonant case.

The analytical expression for the system energy is of interest in the case of large quantum numbers $(n \gg 1)$ which are essential when considering the interaction of the atom with an intensive electromagnetic field. In this limit the matrix elements of the operator $\hat{S}$ are essentially simplified. Using (see HTF 1953)

$$
L_{n}^{\alpha}(x)=\frac{1}{\sqrt{\pi}} \mathrm{e}^{\frac{1}{2} x} x^{-\frac{1}{2} \alpha-\frac{1}{4}} n^{\frac{1}{2} \alpha-\frac{1}{4}} \cos \left[2 \sqrt{n x}-\frac{\alpha \pi}{2}-\frac{\pi}{4}\right]+\mathrm{O}\left(n^{\frac{1}{2} \alpha-\frac{3}{4}}\right)
$$

one may find

$S_{k n}(f)=\frac{(-1)^{n}}{\sqrt{2 \pi f \sqrt{n}}} \cos \left(4 f \sqrt{n}-\frac{(k-n) \pi}{2}-\frac{\pi}{4}\right) \quad n \gg 1 \quad(k-n) \ll n$.

As a result, formula (22) transforms as follows:

$$
\mathcal{E}_{n p}^{(0)}=n-f^{2}+\frac{q E}{2 \sqrt{2 \pi f \sqrt{n}}} \cos \left(4 f \sqrt{n}-\frac{\pi}{4}\right)
$$

and can be used in the limit when the field is described semiclassically.

\section{Time evolution of the system}

The efficiency of the numerical solution is very important for the problem of system time evolution when many stationary states have to be calculated. Therefore in the last section we consider briefly the time evolution of some initial states of the system when the exact solution is used instead of RWA.

Let us suppose, for example, that at the moment 0 the atom occupies an excited state and the quantum field is in the vacuum state. It means that the initial wavepacket in the system considered has the following form:

$$
|\Psi(0)\rangle=|0\rangle \chi_{\uparrow} .
$$

One can expand it in the exact stationary states (8) and calculate the probability $P_{\uparrow}(t)$ of finding the atom in the excited state again at the moment $t$ regardless of the field quantum number. It is well known that in the limits of the Jaynes-Cummings model the value $P_{\uparrow}(t)$ oscillates periodically at the Rabi frequency. The exact expression for $P_{\uparrow}(t)$ is the following

$$
\begin{aligned}
& P_{\uparrow}(t)=\frac{1}{2} \sum_{m p} \sum_{n q} A_{m p} A_{n q} \cos \left[\left(\mathcal{E}_{m p}-\mathcal{E}_{n q}\right) t\right] \sum_{k}\left(C_{k+}^{m p}+C_{k-}^{m p}\right)\left(C_{k+}^{n q}+C_{k-}^{n q}\right) \\
& C_{k-}^{m p}=\sum_{l} S_{k l}(f) C_{l+}^{m p} \\
& A_{m p}=\frac{1}{N \sqrt{2}} \mathrm{e}^{-\frac{1}{2} f^{2}} \sum_{k} \frac{f^{k}}{\sqrt{k !}}\left(C_{k+}^{m p}+C_{k-}^{m p}\right) \\
& N^{2}=\sum_{k}\left[\left(C_{k+}^{m p}\right)^{2}+\left(C_{k-}^{m p}\right)^{2}\right] .
\end{aligned}
$$




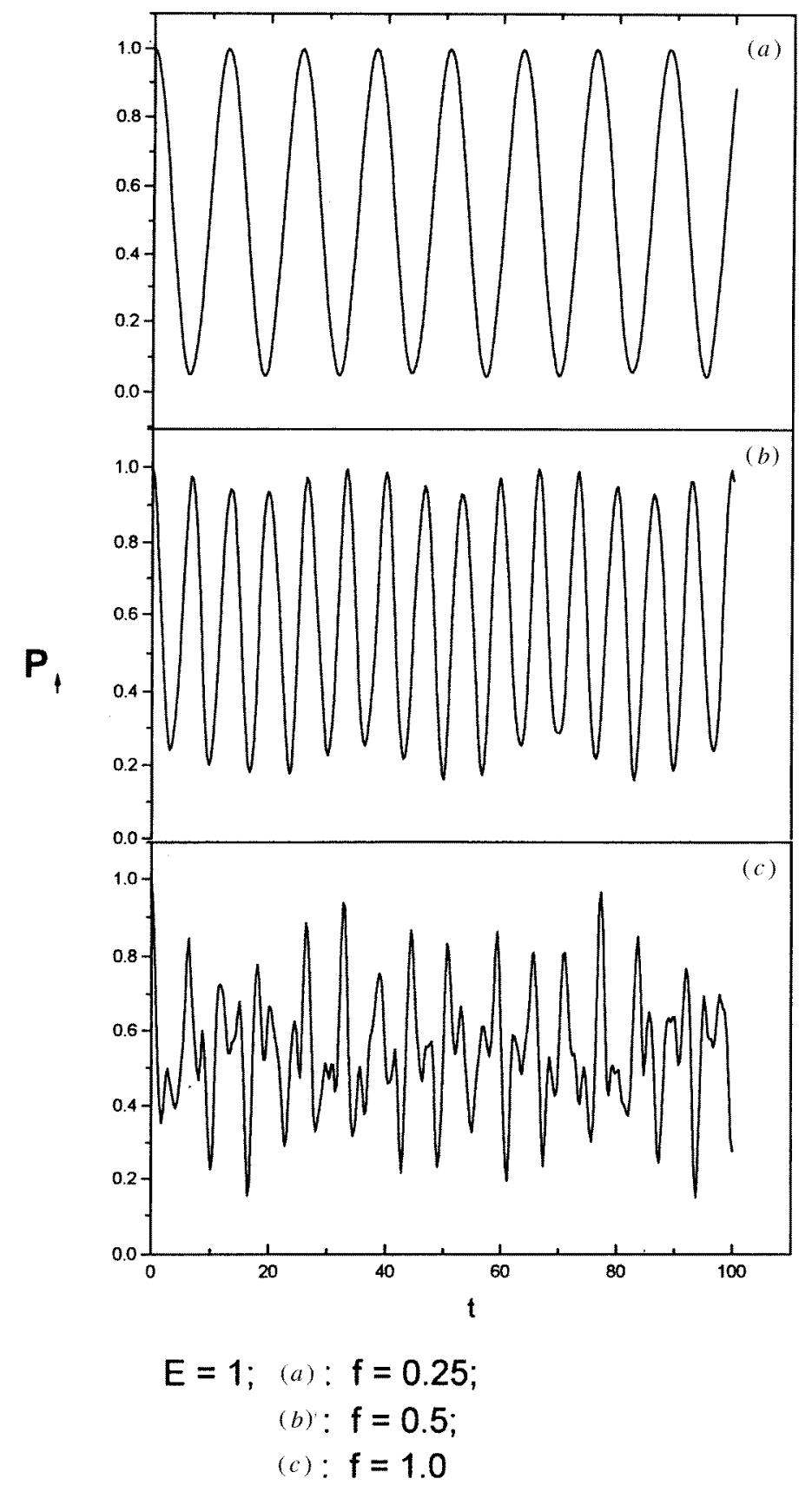

Figure 4. Probability of the population of the atom excited state as a function of time.

We calculated the required probability on the basis of formula (27) and the numerical solution of equations (9) for the stationary states. Figure 4 shows the results of the calculation for various values of the coupling constant. One can see that the increase of the coupling constant leads to modification of the oscillating regime of the function $P_{\uparrow}$ from 


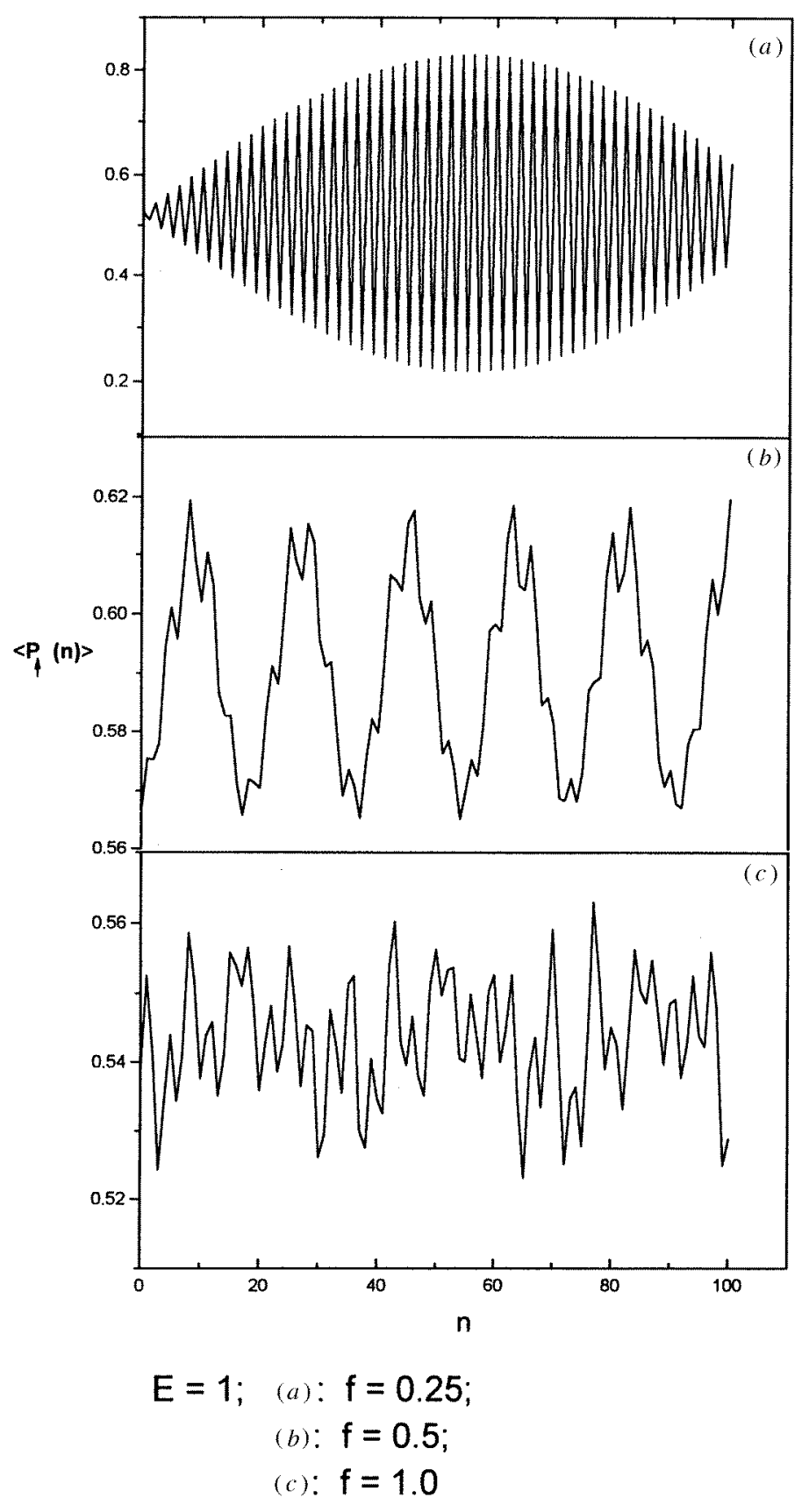

Figure 5. Probability of the excited state population averaged on the main period.

periodic with period $2 \pi$ to the specific case which corresponds to the quasi-periodic motion in the theory of instability (e.g. Richtmyer 1981) and arises as a result of the superposition of many motions with incommensurable frequencies.

The same tendency can be observed in figure 5 which shows the function $\left\langle P_{\uparrow}(n)\right\rangle$ 


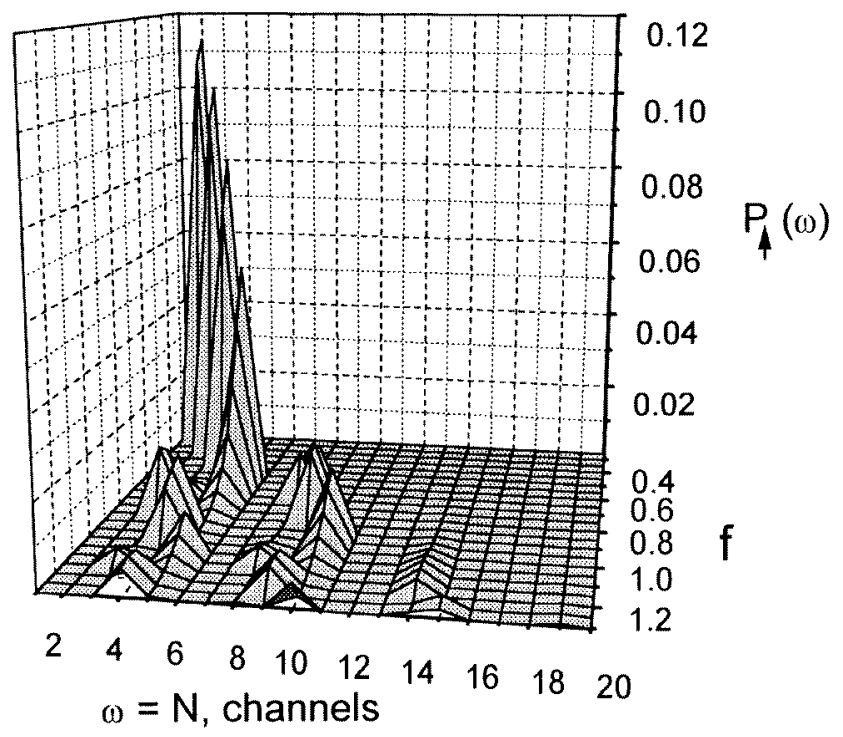

Figure 6. Spectral function for the probability of the excited state population.

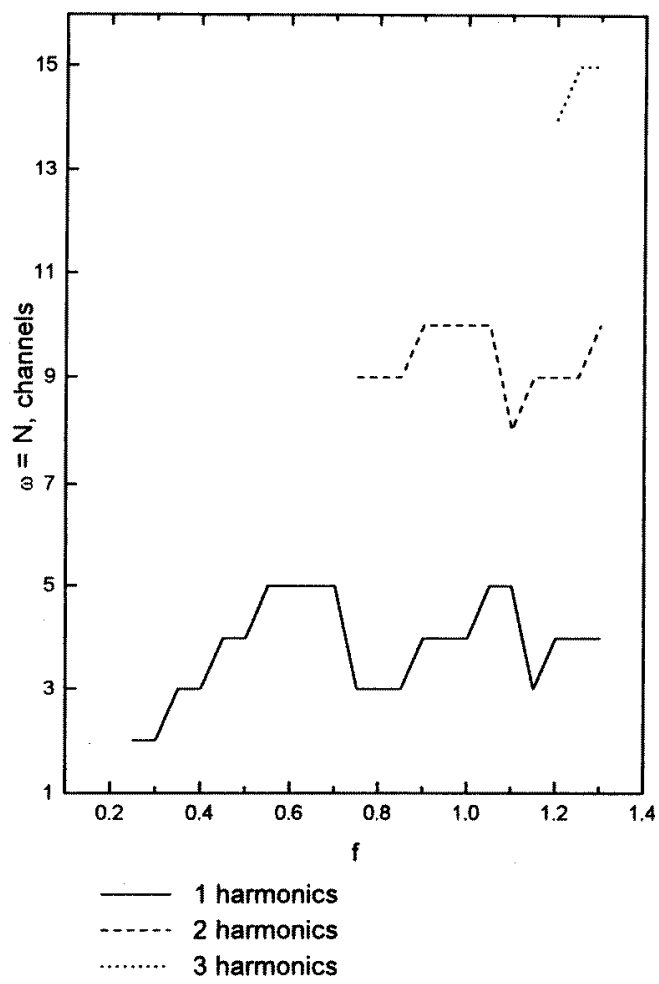

Figure 7. Amplitudes of the main harmonics as functions of the coupling constant.

averaged over the main period of the oscillation, that is

$$
\left\langle P_{\uparrow}(n)\right\rangle=\frac{1}{2 \pi} \int_{2 \pi n}^{2 \pi(n+1)} \mathrm{d} t P_{\uparrow}(t) .
$$


It is known that one of the possible ways of chaotization in dynamical systems is connected with doubling of the oscillation frequency (Richtmyer 1981). One can see (figures 6 and 7) that such behaviour appears in the system considered. In particular, figure 6 shows the spectrum of the function $P_{\uparrow}(t)$, i.e. the function

$$
P_{\uparrow}(\omega)=\int_{-\infty}^{\infty} \mathrm{d} t \mathrm{e}^{-\mathrm{i} \omega t} P_{\uparrow}(t)
$$

depending on the coupling constant $f$.

Figure 7 demonstrates the non-monotonic dependence of the main harmonic amplitudes on the value $f$.

\section{Conclusion}

We have considered the solution to the Schrödinger equation for a two-level system in a one-mode quantum field on the basis of the operator method. It has permitted us to build an effective algorithm for finding a numerical solution with arbitrary accuracy and to find an obvious representation of the system energy spectrum in all parameter regions. Also, the analytical approximate formulae are derived for the energy spectrum. These formulae describe the right energy level sequence with respect to an exact integral of motion.

The particular interest is in peculiarities of the time evolution of the TLS which are stipulated by accounting for the counter-rotating terms in the Hamiltonian. It is shown that these peculiarities can be described with high accuracy on the basis of the presented numerical solution. However, the detailed analysis of these peculiarities will be carried out in following papers.

\section{References}

Allen L and Eberly J 1975 Optical Resonance and Two Level Atoms (New York: Wiley)

Feranchuk I and Komarov L 1982 Phys. Lett. 88A 212

1984 J. Phys. A: Math. Gen. 173111

Feranchuk I, Komarov L and Nichipor I 1987 J. Phys. A: Math. Gen. 203849

Feranchuk I, Komarov L, Nichipor I and Ulyanenkov A 1995 Ann. Phys., NY 238370

Graham R and Hoehnerbach M 1984 Phys. Lett. 101A 61

HTF 1953 High Transcendental Functions (New York: McGraw-Hill)

Jaynes E and Cummings F 1963 Proc. IEEE 511963

Kus M and Lewenstein M 1986 J. Phys. A: Math. Gen. 19305

Lais P and Steimle T 1990 Opt. Commun. 78346

Meystre P and Steimle T 1982 Phys. Lett. 89A 390

Reik H and Doucha M 1986 Phys. Rev. Lett. 57787

Reik H, Lais P, Stüzle M E and Doucha M 1987 J. Phys. A: Math. Gen. 206327

Reik H, Nusser H and Ribeiro A 1982 J. Phys. A: Math. Gen. 153491

Richtmyer R 1981 Principles of Advanced Mathematical Physics vol 2 (New York: Springer) 